07

\title{
Карты широкополосной квантовой памяти на частотной гребенке атомных линий*
}

\author{
(C) Н.М. Арсланов, С.А. Моисеев व \\ Казанский квантовый центр, \\ Казанский национальный исследовательский технический университет имени А.Н. Туполева, \\ 420126 Казань, Россия \\ ฯ e-mail: samoi@yandex.ru
}

Поступила в редакцию 24.09.2018 г.

Исследовано влияние параметров частотной гребенки неоднородного уширения атомных линий на эффекты дисперсии в протоколе оптической квантовой памяти, реализуемого на такой атомной системе. Полученные результаты позволили построить карты дисперсии и квантовой эффективности для реализации изучаемого протокола на кристаллах с редкоземельными ионами, перспективного для создания оптического квантового репитера.

DOI: $10.21883 /$ OS.2019.01.47050.269-18

\section{Введение}

Широкополосная и многокубитовая квантовая память является одним из ключевых элементов для создания квантового репитера и универсального квантового компьютера [1]. В настоящее время среди разрабатываемых подходов к оптической квантовой памяти большое внимание уделяется квантовой памяти на модифицированных протоколах фотонного эха, приспособленных к реализации обратимого взаимодействия света с оптически плотными когерентными атомными ансамблями [2-11], которые являются перспективными для запоминания большого числа фотонных кубитов [4,5]. На основе подхода [3], обеспечивающего точное обращение временной динамики сигнального поля в излучении сигнала фотонного эха, был предложен ряд модификаций [6-10], которые значительно расширили возможности экспериментальной реализации квантовой памяти на фотонном эхе. Простота реализации АЧГ-протокола (АЧГ — атомно частотная гребенка от английского „,atomic frequency comb“, AFC) и его перспективные потенциальные возможности определили значительный интерес к его разработке. К настоящему времени в данном протоколе достигнута точность восстановления состояния однофотонных полей более 90\% [6] и показана возможность сохранения 64 предельно слабых световых импульсов со средним числом фотонов в импульсе $\bar{n}=0.5$ [4] и даже 1060 световых импульсов [11]. Кроме того, недавно продемонстрировано сохранение перепутанных состояний фотонов [12] и временных фотонных кубитов в 26 мультиплексированных спектральных модах [13]. Наконец, АЧГ-протокол был успешно применен для работы с многочастотными каналами записи [14].

К настоящему времени наилучшая квантовая эффективность, равная 35\%, была достигнута эксперименталь-

* XIII International Conference on Hole Burning, Single Molecule, and Related Spectroscopies: Science and Applications (HBSM-2018), August 6-12, 2018, Suzdal-Moscow, Russia. но в 2009 году [12], и пока отсутствует ясное понимание путей дальнейшего повышения эффективности в этом протоколе. Помещение атомов в оптический резонатор позволило достичь большей квантовой эффективности, где, однако, затруднительно запоминание широкополосных световых сигналов, как это может иметь место на атомных ансамблях, находящихся вне оптического резонатора, что делает актуальным дальнейшую разработку широкополосного АЧГ-протокола без использования резонаторов.

В настоящей работе мы исследуем влияние спектральных параметров АЧГ-структуры и ширины линии на дисперсионные эффекты, проявляющиеся в изучаемом протоколе памяти, и показываем, каким образом можно эффективнее сохранять широкополосные световые поля за счет создания АЧГ-структуры в оптимальном спектральном интервале $\Delta_{0}$ внутри естественного неоднородного уширения оптического перехода. Таким образом, мы также нашли возможность значительного увеличения рабочего спектральный интервал $\Delta_{q m}$, в котором реально достижение высокой квантовой эффективности для запоминания оптических импульсов произвольной формы.

\section{Частотная дисперсия в АЧГ-протоколе}

В АЧГ-протоколе [6,7] используются атомные системы, в которых неоднородное уширение $\Delta_{i n}$ оптического перехода на частоте $\omega_{31}$ модифицируется в виде дискретной структуры узких линий с шириной $\gamma$ и частотным интервалом $\Delta$ между двумя ближайшими линиями (рис. $1, a)$. Такая структура атомных линий часто реализуется путем спектрально селективного возбуждения атомов на другие уровни энергии [11]. При сохранении сигнального светового поля в атомной системе происходит возбуждение неравновесной поляризации $P(t)$, которая при дальнейшей эволюции автоматически вызывает 




Рис. 1. (a) АЧГ-структура на неоднородном уширении рабочей линии в кристалле. Гребенки структуры заполняют равномерно всю ширину перехода. $(b)$ АЧГ-структуры, приготовленные в узкой части неоднородно уширенной линии $\Delta_{\text {in }}$.

излучение сигнала эха через время $\tau=2 \pi / \Delta$. В ,прямой“ АЧГ-схеме эксперимента, когда сигнал эха излучается со-направленно начальному импульсу, максимальный теоретический предел эффективности равен 54\%, который может быть достигнут при достаточно большой оптической плотности атомного перехода $\left(\alpha_{0} L>100\right)$ и резкости атомных линий $f=\Delta / \gamma>50$.

Дальнейшее повышение квантовой эффективности подразумевает переход к „обращенной“ АЧГ-схеме, когда сигнал эха распространяется в противоположном направлении. Теоретический анализ квантовой эффективности в обратной схеме АЧГ-протокола с бесконечно широкой линией атомного перехода дает зависимость [7]:

$$
\eta \cong\left(1-e^{-\alpha_{0} L / f}\right)^{2} e^{-7 / f^{2}}
$$

которая указывает на возможность достижения квантовой эффективности, близкой к $100 \% \quad(\eta \sim 0.999$, $\left.\alpha_{0} L \sim 870, f \sim 93\right)$, в случае, когда ширина сигнального импульса $\delta \omega_{\mathrm{ph}}$ достаточно узкая по сравнению со спектральной шириной АЧГ-структуры, $\Delta_{0} \gg \delta \omega_{\mathrm{ph}}$. Для практического применения в квантовом репитере достаточно иметь эффективность $\eta=90 \%$, что может быть реализовано в „обращенной“ АЧГ-схеме уже при относительно умеренной оптической плотности $\alpha_{0} L \sim 40$, $f \sim 10[7,15]$.

Обсуждение возможностей экспериментальной peaлизации АЧГ-протокола часто проводится на основе использования формулы (1), которая указывает на необходимость использования только большой оптической плотности неоднородно уширенного резонансного перехода. При этом сигнальные поля могут иметь спектральную ширину $\delta \omega_{\text {ph }}$, сопоставимую с шириной АЧГструктуры $\Delta_{0}, \Delta_{0} \sim \delta \omega_{\mathrm{ph}}$. В этом случае параметры восстановленного импульса света

$$
A_{\text {out }}(\tau) \sim \int d \omega \Gamma(\omega) A_{\text {in }}(\omega) \exp (-i \omega \tau)
$$

определяются спектральной функцией

$$
\Gamma(\omega)=\frac{1-\exp \left(i \omega_{31} \chi(\omega) L / c\right)}{1-i \operatorname{Re} \chi(\omega) / \operatorname{Im} \chi(\omega)},
$$

в которой заметную роль начинает играть спектральная дисперсия среды, определяемая параметрами АЧГструктуры [16]:

$$
\chi(\omega)=-\frac{c}{\omega} \alpha_{0} \exp \left(-\xi \frac{\omega^{2}}{\Delta_{\text {in }}^{2}}\right)\left(\operatorname{Erfi}\left(\sqrt{\zeta} \frac{\omega}{\Delta_{\text {in }}}\right)+i\right),
$$

где $\xi=4 \operatorname{Ln} 2, c-$ скорость света, $G(\omega)=\sqrt{\frac{\xi}{\pi}} \frac{1}{\Delta_{\text {in }}} \times$ $\times \exp \left(-\xi \frac{\omega^{2}}{\Delta_{\text {in }}^{2}}\right)-$ распределение неоднородного уширения линии.

В реальных экспериментах АЧГ-структура с шириной $\Delta_{0}$ занимает лишь часть неоднородного уширения $\Delta_{\text {in }}$ (рис. $1, b$ ). Таким образом, частотное распределение атомов среды можно учитывать в виде

$$
C(\omega)=G(\omega)(1-\Pi(\omega))+G(\omega) \Pi(\omega) \sum_{n} k(\omega-\Delta n),
$$

где $\Pi(\omega)=\Theta\left(\omega+\frac{\Delta_{0}}{2}\right)-\Theta\left(\omega-\frac{\Delta_{0}}{2}\right), \Theta(\omega)=1(\omega \geq 0)$, $0(\omega<0)-$ функция ступеньки, $k(\omega)$ - распределение отдельного зубца гребенки АЧГ-структуры, суммирование идет по всем зубцам гребенки в пределах структуры АЧГ. Дисперсию такой среды будет определять уже не только АЧГ-структура

$$
\operatorname{Re} \chi_{A F C}(\omega) \sim \sum_{n} \mathrm{P} \int_{-\Delta_{0} / 2}^{\Delta_{0} / 2} d \omega^{\prime} \frac{G(\omega) k(\omega-\Delta n)}{\omega-\omega^{\prime}},
$$



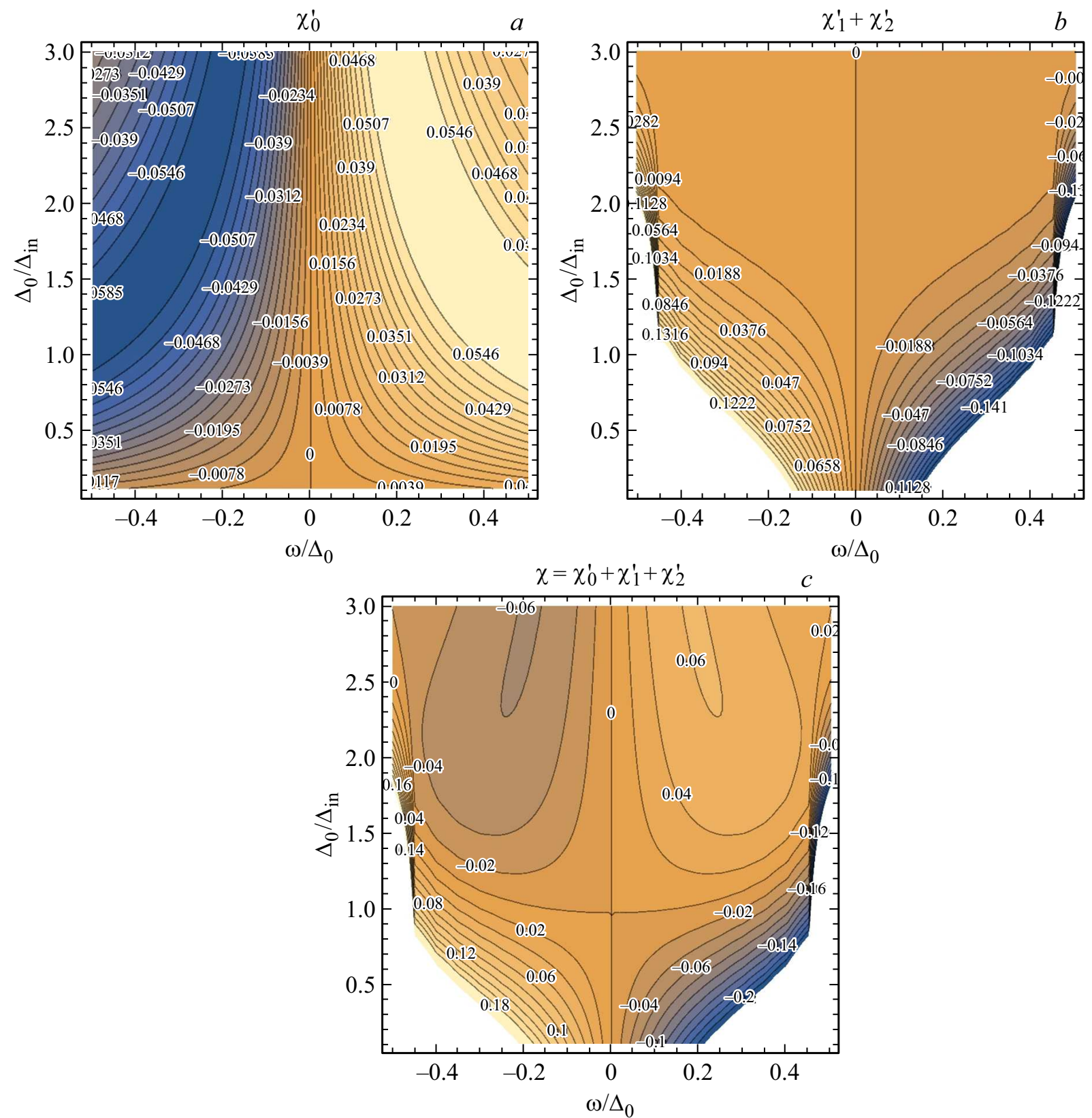

Рис. 2. Контурные карты проницаемости: (a) проницаемость, обусловленная АЧГ-структурой, в зависимости от ширины структуры $\Delta_{0} / \Delta_{\text {in }}$ и частоты $\omega$ в пределах этой структуры; $(b)$ поведение проницаемости, обусловленной крыльями неоднородного уширения, в зависимости от ширины структуры $\Delta_{0} / \Delta_{\text {in }}$ и частоты $\omega$ в пределах этой структуры; $(c)$ поведение суммарной проницаемости от центральной части АЧГ-структуры и ее крыльев в зависимости от ширины структуры $\Delta_{0} / \Delta_{\text {in }}$ и частоты $\omega$ в пределах этой структуры. Из-за дисперсии существует область, где противоположные вклады подавляют друг друга, и общая дисперсия меняется очень плавно.

(здесь идет суммирование по гребенкам решетки в пределах неоднородного уширения), но и боковые крылья неоднородного уширения

$$
\operatorname{Re} \chi_{\text {wings }}(\omega) \sim \mathrm{P} \int_{-\infty}^{-\Delta_{0} / 2} d \omega^{\prime} \frac{G(\omega)}{\omega-\omega^{\prime}}+\mathrm{P} \int_{\Delta_{0} / 2}^{\infty} d \omega^{\prime} \frac{G(\omega)}{\omega-\omega^{\prime}},
$$

атомы которых не подвергались предварительному воздействию при приготовлении АЧГ-структуры.
На рис. 2 приведено сравнение различных вкладов в дисперсию в зависимости от размера АЧГ-структуры $\Delta_{0} / \Delta_{\text {in }}$ и отстройки частоты от рабочей частоты в пределах этой структуры. Вклад в дисперсию от АЧГ-структуры имеет вид нормальной дисперсии (рис. 2,a) в центре линии, где $\chi \sim \omega$. Максимальное изменение дисперсии происходит вблизи центра неоднородного уширения вблизи нулевой частотной отстройки. При этом влияние „крыльев“ неоднородного уширения приводит к 


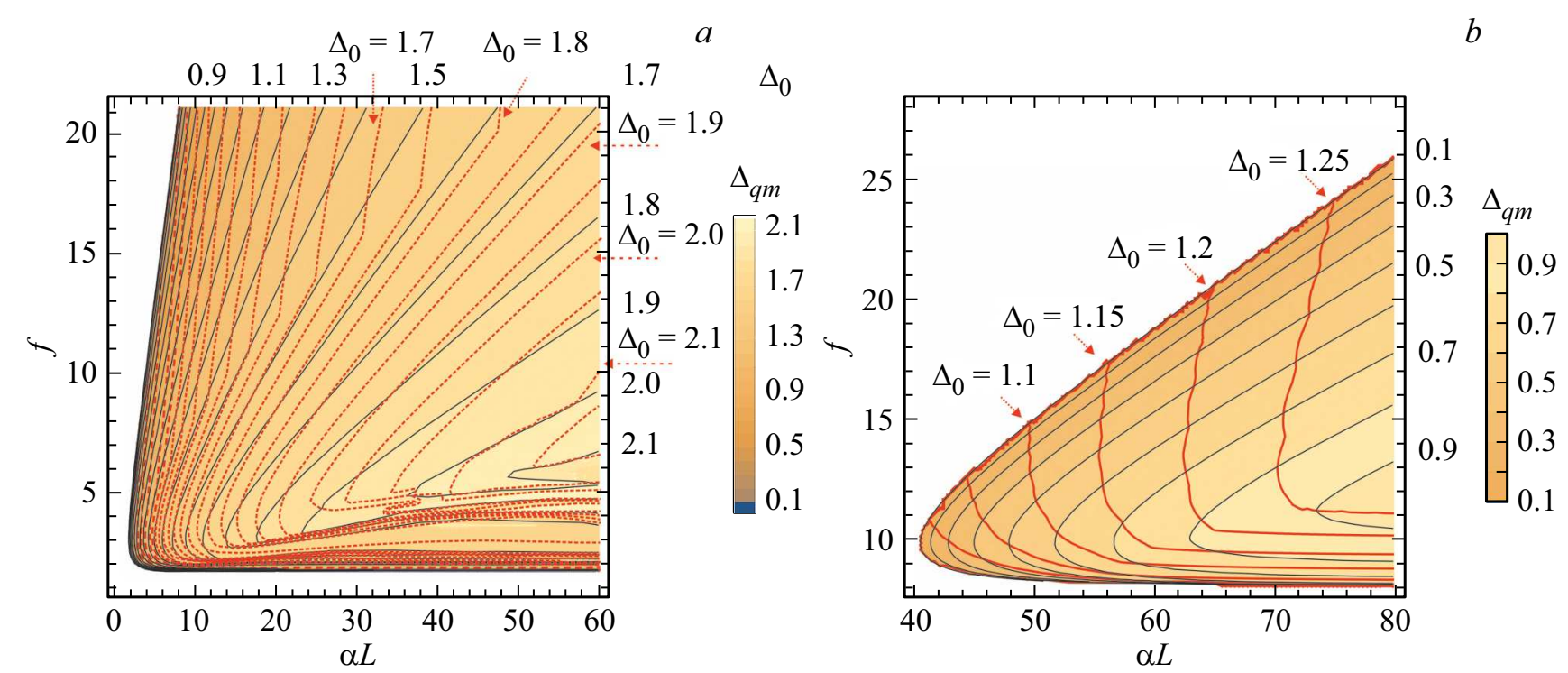

Рис. 3. Карта максимальной ширины $\Delta_{q m}$ при оптимальной $\Delta_{0}$ в единицах $\Delta_{\text {in. }}$ Красными линиями обозначены изолинии

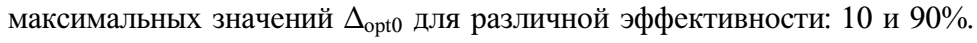

аномальной дисперсии (рис. 2, b). Резкое изменение дисперсии имеет место при достаточно малых спектральных размерах АЧГ-структуры $\Delta_{0}$, формируемой вблизи центра линии, а при увеличении $\Delta_{0}$ резкие изменения дисперсии в основном проявляются на границах АЧГструктуры. Влияние этих двух разнонаправленных вкладов приводит к тому, что при определенных спектральных параметрах АЧГ-структуры и резкости $f$ происходит взаимная компенсация противоположных дисперсионных эффектов от центральной части и ее „крыльев“, что проявляется в возникновении спектральной области с подавленной дисперсией (рис. 2,c). Наличие такой широкой области с подавленной дисперсией определяет максимальную ширину импульса, который может сохраняться в памяти без существенных искажений [17].

\section{Эффективность широкополосного АЧГ-протокола}

Кроме практического достижения максимально возможной рабочей [17] спектральной ширины АЧГпротокола $\Delta_{q m}$ также важно, чтобы восстановление сигнального импульса в этом спектральном диапазоне происходило при достаточно высокой эффективности. Таким образом, задавая требуемый уровень эффективности $\eta(\omega)$ (которая зависит от величины поглощения $\operatorname{Im} \chi(\omega))$, необходимо определить максимальную достижимую ширину квантовой памяти и ширину АЧГ-структуры, при которых не происходит существенного затухания восстановленного светового сигнала из-за нарушения фазового синхронизма, вызванного частной дисперсией (благодаря $\operatorname{Im} \chi(\omega)$ и $\operatorname{Re} \chi(\omega)$ ). Используя полученные нами выражения, мы провели численное моделирование такой задачи по поиску оптимальных параметров квантовой памяти на АЧГ-структуре. На рис. 3 представлены карты расчета максимальной ширины квантовой памяти $\Delta_{q m}$ от величины $\Delta_{0}$ АЧГ-структуры для двух значений эффективности 10 и $90 \%$ в зависимости от оптической плотности $\alpha_{0} L$ и резкости структуры $f=\Delta / \gamma$. Полученные данные приведены с учетом конечной ширины световых импульсов. Отметим, что данные карты построены в относительных единицах, что позволяет их использовать для различных кристаллов, в которых величина неоднородного уширения может существенно различаться.

Для случая относительно малой эффективности (рис. $3, a) \quad 10 \%$ спектральная ширина АЧГ-структуры занимает большую часть спектра неоднородного уширения. Поэтому при такой малой эффективности спектральный диапазон квантовой памяти также имеет большую ширину. Для увеличения эффективности необходимо уменьшать спектральную ширину $\Delta_{0}$ АЧГ-структуры для усиления вклада „крыльев“ линии оптического перехода в общую спектральную дисперсию в центре линии. Например, для достижения эффективности 90\% при использовании $\alpha_{0} L \sim 40, f \sim 10$ из формулы (1) имеем минимальную ширину АЧГ-структуры порядка $\Delta_{0} \sim \Delta_{\text {im }}$, при этом рабочий спектральный диапазон квантовой памяти $\Delta_{q m} \sim 0.1 \Delta_{\text {in. }}$. При увеличении оптической плотности и резкости мы переходим в область больших значений спектральной ширины квантовой памяти. Например, при $\alpha_{0} L \sim 80, f \sim 10$ ширина АЧГ-структуры $\Delta_{0} \sim 1.25 \Delta_{\text {in }}$, а ширина рабочего диапазона квантовой памяти возрастает до $\Delta_{q m} \sim 0.9 \Delta_{\text {in }}$, что позволит сохранять достаточно большое число импульсов, необходимое для использования в квантовых репитерах. 


\section{Выводы}

Мы исследовали влияние параметров периодической частотной гребенки атомных линий на свойства АЧГ-протокола квантовой памяти. Показаны способы выбора оптимальных значений спектральной ширины АЧГ-структуры для управления рабочим диапазоном квантовой памяти и достижения высокой квантовой эффективности. С этой целью построены наглядные детальные спектральные карты, впервые позволяющие определять реальные способы реализации квантовой эффективности 90\% за счет выбора параметров оптического квантового перехода: резкости частной структуры линии, спектральной ширины и оптической плотности резонансного перехода. Полученные результаты указывают на возможности реализации схем высокоэффективной широкополосной квантовой памяти на кристаллах с редкоземельными ионами, перспективной для использования в оптическом квантовом репитере.

Работа поддержана Российским научным фондом в рамках гранта № 14-12-01333-П.

\section{Список литературы}

[1] Sangouard N., Simon C., De Riedmatten H. et al. // Rev. Mod. Phys. 2011. V. 83. P. 33.

[2] Lvovsky A.I., Sanders B.C., Tittel W. // Nature Photonics 2009. V. 3. P. 706.

[3] Moiseev S.A., Kröll S. // Phys. Rev. Lett. 2001. V. 87. P. 173601.

[4] Tittel W., Afzelius M., Chaneliére T. et al. // Laser Photon. Rev. 2010. V. 267. P. 244.

[5] Usmani I., Afzelius M., de Riedmatten H. et al. // Nat. Commun. 2010. V. 1. P. 12.

[6] De Riedmatten H., Afzelius M., Staudt M.U. et al. // Nature 2008. V. 456. P. 773.

[7] Afzelius M., Simon C., De Riedmatten H. et al. // Phys. Rev. A. 2009. V. 79. P. 1.

[8] Alexander A.L., Longdell J.J., Sellars M.J. et al. // Phys. Rev. Lett. 2006. V. 96. P. 043602.

[9] Damon V., Bonarota M., Louchet-Chauvet A. et al. // New J. Phys. 2011. V. 13. P. 093031.

[10] McAuslan D.L., Ledingham P.M., Naylor W.R. et al. // Phys. Rev. A. 2011. V. 84. P. 022309.

[11] Bonarota M., Le Gouët J.-L., Chaneliére T. // New J. Phys. 2011. V. 13. P. 013013.

[12] Saglamyurek E., Sinclair N., Jin J. et al. // Nature. 2011. V. 469. P. 512.

[13] Sinclair N., Saglamyurek E., Mallahzadeh H. et al. // Phys. Rev. Lett. 2014. V. 113. P. 053603.

[14] Saglamyurek E., Puigibert M.G., Zhou Q. et al. // Nature Communications. 2016. V. 7. P. 11202.

[15] Amari A., Walther A., Sabooni M. et al. // J. Lumin. 2010. V. 130. P. 1579.

[16] Moiseev S.A., Le Gouët J.-L. // J. Phys. B: Atomic, Molecular and Optical Physics. 2012. V. 45. P. 124003.

[17] Arslanov N.M., Moiseev S.A. // Quantum Electron. 2017. V. 47. P. 783. 